\title{
Fortificação das farinhas com ferro e controle da anemia em gestantes de Teresina, Piauí, Brasil
}

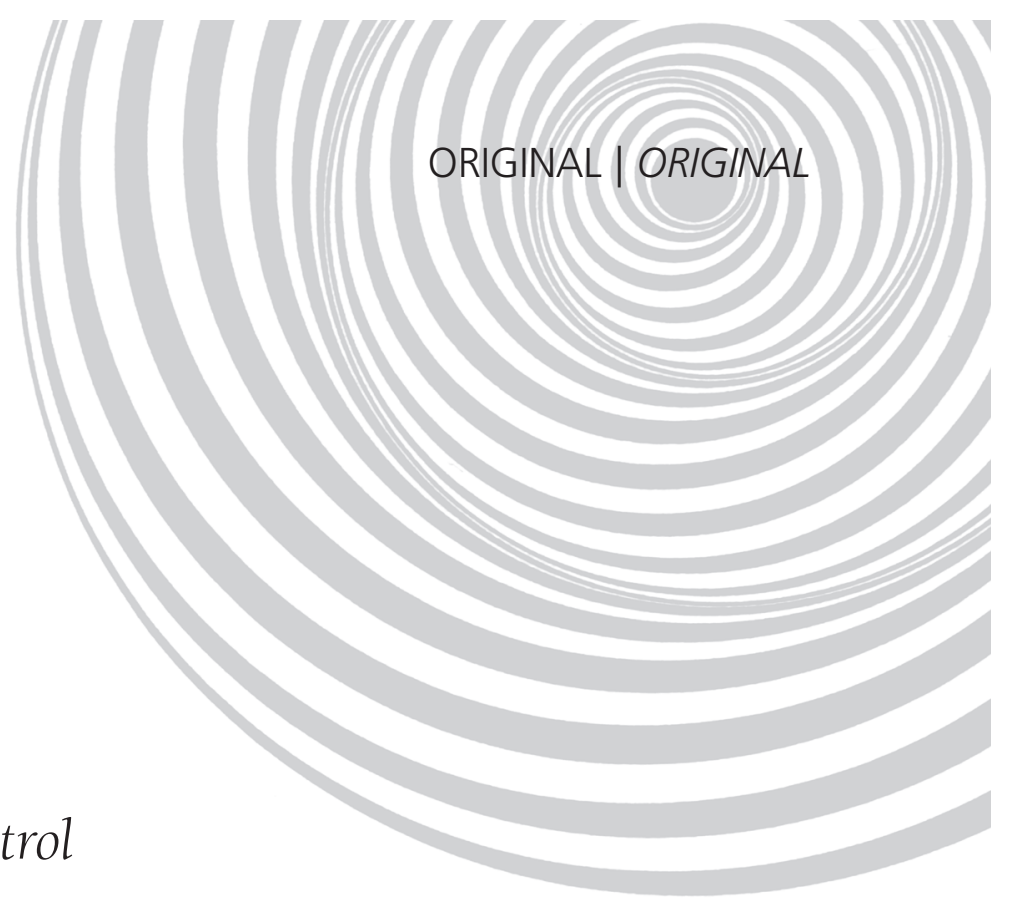

\section{Fortification of flours with iron and control \\ of anemia in pregnant women in}

Teresina, Piauí, Brazil

Manoel Dias de SOUZA FILHO ${ }^{1}$

Clênia Vanessa Ximenes DAMASCENO²

Sophia Cornbluth SZARFARC ${ }^{3}$

Elizabeth FUJIMORI ${ }^{4}$

Marcos Antônio de Mota ARAÚJO ${ }^{5}$

Regilda Saraiva dos Reis MOREIRA-ARAÚJO²

\section{RE S U M O}

\section{Objetivo}

Comparar níveis de hemoglobina e ocorrência de anemia em gestantes antes e depois da fortificação das farinhas e caracterizar a prática alimentar de gestantes usuárias atuais dos serviços.

\section{Métodos}

Estudo transversal que analisou dados de hemoglobina de gestantes atendidas em dois serviços públicos de maior demanda pré-natal do Município de Teresina, Piauí. Os dados foram obtidos de 854 prontuários de gestantes distribuídas em dois grupos: não fortificado (427 gestantes com parto anterior a junho/2004) e fortificado (427 gestantes com data da última menstruação posterior a junho/2005). Gestantes com nível de hemoglobina $<11,0 \mathrm{~g} / \mathrm{dL}$ foram consideradas anêmicas. O consumo alimentar foi analisado por meio de questionário de frequência alimentar semiquantitativo.

\section{Resultados}

Níveis médios de hemoglobina aumentaram significativamente de 11,7g/dL, DP=1,2 para 12,4g/dL, DP=1,3 $(p<0,001)$ após a fortificação. A prevalência de anemia caiu de $27,2 \%$ no grupo não fortificado para $11,5 \%$ no grupo fortificado $(p<0,001)$.

\footnotetext{
$\overline{\mathbf{1}}$ Universidade Federal do Piauí, Coordenação de Biomedicina. Parnaíba, PI, Brasil.

2 Universidade Federal do Piauí, Departamento de Nutrição. Campus Ministro Petrônio Portela, SG 13, Ininga, 64049-550,

Teresina, PI, Brasil. Correspondência para/Correspondence to: R.S.R. MOREIRA ARAÚJO. E-mail: <regilda@ufpi.br>.

3 Universidade de São Paulo, Faculdade de Saúde Pública, Departamento de Nutrição. São Paulo, SP, Brasil.

${ }^{4}$ Universidade de São Paulo, Departamento de Enfermagem em Saúde Coletiva. São Paulo, SP, Brasil.

5 Fundação Municipal de Saúde, Gerência de Planejamento. Teresina, PI, Brasil.
} 
680 | M.D. SOUZA FILHO et al.

\section{Conclusão}

Houve melhora significativa no quadro de anemia após a fortificação das farinhas, o que sugere que a intervenção foi efetiva no controle da deficiência de ferro, porém o estudo não permite atribuir tal resultado apenas à implantação dessa medida, embora análise da prática alimentar tenha mostrado que as gestantes de Teresina (PI) apresentaram condição diferenciada de alimentação, com consumo frequente de fontes naturais de ferro e de facilitadores de sua absorção, além de alimentos fortificados.

Termos de indexação: Anemia. Cuidado pré-natal. Gravidez. Hemoglobina.

\section{A B S T R A C T}

\section{Objective}

This study compared the hemoglobin levels and the occurrence of anemia in pregnant women before and after the fortification of flours and characterized the eating habits of pregnant women who currently use the service.

\section{Methods}

This cross-sectional study analyzed the hemoglobin data of pregnant women seen by two public services with greater prenatal demand in the city of Teresina, Piauí. The data were obtained from 854 medical records of the women distributed into two groups: not fortified (427 women who would deliver before June 2004) and fortified (427 women whose last menstruation was after June 2005). Women with a hemoglobin level $<11.0 \mathrm{~g} / \mathrm{dL}$ were considered anemic. Food intake was analyzed by a semiquantitative food frequency questionnaire.

\section{Results}

Mean hemoglobin levels increased significantly from $11.7 \mathrm{~g} / \mathrm{dL}, S D=1.2$, to $12.4 \mathrm{~g} / \mathrm{dL}, S D=1.3(p<0.001)$ after fortification. The prevalence of anemia decreased from $27.2 \%$ in the non-fortified group to $11.5 \%$ in the fortified group $(p<0,001)$.

\section{Conclusion}

Anemia improved significantly after fortification of the flours, suggesting that the intervention effectively controlled iron deficiency. However, it is not possible to attribute the improved iron levels on this measure alone, even though analysis of their eating habits showed that pregnant women from Teresina (PI) presented unusual food habits, with frequent consumption of natural iron sources and foods that facilitate its absorption, in addition to the fortified foods.

Indexing terms: Anemia. Prenatal care. Pregnancy. Hemoglobin.

\section{N T R O D U Ç Ã O}

A anemia por deficiência de ferro é a desordem nutricional mais comum, de forma que se têm tornado repetitivas as estimativas da Organização Mundial de Saúde (OMS) de que mais de dois bilhões de pessoas no mundo são anêmicas ${ }^{1,2}$.

Dentre os grupos de risco, as gestantes merecem destaque especial, pois durante a gravidez há uma elevada demanda de ferro em um curto período de tempo ${ }^{1,3}$. Ademais, em um percentual elevado de mulheres em idade reprodutiva, a reserva do mineral é baixa ou inexistente, condição que dificulta o suprimento da elevada demanda gestacional ${ }^{4,5}$. Justifica-se, assim, a denominação "grupo vulnerável indicador" atribuída às gestantes pela OMS, de forma que o conhecimento da prevalência de anemia nesse grupo permite identificar o risco dessa deficiência na população de gestantes.

Em termos de saúde pública, a relevância da prevenção da anemia na gestação decorre principalmente dos efeitos deletérios que ocasiona na saúde do binômio mãe e filho, uma vez que a anemia associa-se a maior risco de prematuridade, baixo peso ao nascer, mortalidade perinatal, além de maior risco de deficiência de ferro no recém-nascido 6 . 
A alimentação pobre em ferro e/ou com baixo potencial de absorção do mineral é o principal determinante da anemia, de forma que todos os membros de uma comunidade que se alimentam com tal dieta são vítimas potenciais da deficiência. Assim, os programas de intervenção para o controle da deficiência de ferro que visam a aumentar o aporte do mineral devem ser abrangentes para atender toda a população'.

Por reconhecer que a deficiência de ferro não se restringe a grupos específicos e que a anemia ferropriva representa um problema nutricional importante, com severas consequências econômicas e sociais, o governo brasileiro tornou obrigatória, por decreto ${ }^{7}$, a adição de ferro e de ácido fólico nas farinhas de trigo e milho, a partir de junho de 2004. Essa medida governamental permite garantir uma fonte complementar permanente de ferro para toda a população brasileira e uma perspectiva altamente promissora para diminuir a prevalência da deficiência marcial e controlar a anemia ferropriva.

Nesse contexto, o objetivo do presente estudo foi comparar níveis de Hemoglobina $(\mathrm{Hb})$ e ocorrência de anemia em gestantes antes e depois da fortificação das farinhas com ferro e ácido fólico e caracterizar a prática alimentar de gestantes usuárias atuais dos serviços estudados.

\section{M É T O D O S}

Este estudo fez parte do projeto colaborativo "Impacto da Ingestão de Farinhas de Trigo e de Milho Fortificadas com Ferro e Ácido Fólico na Concentração de Hemoglobina de Gestantes na Rede Pública de Saúde do Brasil", financiado pelo Conselho Nacional de Desenvolvimento Científico e Tecnológico (CNPq) (processo n 402295/ 2005-6).

Foi desenvolvido como um estudo transversal que avaliou níveis de $\mathrm{Hb}$ de gestantes antes e após pelo menos um ano de efetiva implantação da fortificação das farinhas de trigo e milho com ferro e ácido fólico. Os dados foram coletados em dois serviços públicos de maior demanda de atendimento pré-natal do município de Teresina (PI), que representam os dois principais serviços da rede pública do Estado e do Município no que se refere ao atendimento pré-natal e que assistem gestantes com características antropométricas e socioeconômicas semelhantes.

A escolha de gestantes para avaliar a fortificação das farinhas considerou dois aspectos. $\mathrm{O}$ primeiro, já mencionado, refere-se ao fato de as gestantes constituírem o grupo vulnerável indicador da anemia. O segundo, que tornou factível o desenvolvimento deste estudo, é o fato de a dosagem de $\mathrm{Hb}$ ser um dos exames laboratoriais que deve ser solicitado na primeira consulta pré-natal realizada em qualquer serviço público de saúde brasileiro 8 .

Foram analisados 854 prontuários de gestantes distribuídos em dois grupos: Não Fortificado (NF), formado por 427 gestantes que tiveram o parto antes de junho de 2004; e Fortificado (F), composto por 427 gestantes com Data da Última Menstruação (DUM) posterior a junho de 2005. A amostra incluiu somente gestantes de baixo risco obstétrico, cujo prontuário registrava pelo menos a DUM e a data e o resultado do exame de concentração de $\mathrm{Hb}$. Gestantes com concentração de $\mathrm{Hb}$ inferior a $11,0 \mathrm{~g} / \mathrm{dL}$ foram consideradas anêmicas ${ }^{1,9}$.

Com vistas a identificar a prática alimentar, 30 gestantes não envolvidas na amostra, porém usuárias dos serviços de pré-natal estudados, à época da coleta de dados dos prontuários, foram entrevistadas. O consumo alimentar foi analisado por meio de um Questionário de Frequência Alimentar (QFA) semiquantitativo ${ }^{10}$, validado localmente. O QFA incluiu alimentos fontes de ferro naturais ou fortificados e facilitadores da absorção do mineral (frutas cítricas), com frequências categorizadas em: diariamente, 2 a 4 vezes/semana, 1 vez/semana, raramente ou nunca.

Para verificar se houve associações estatísticas, utilizou-se o teste do $\chi^{2}$ entre os trimestres de gestação e os grupos fortificado e não fortificado. O teste $t$ de Student foi aplicado entre os trimestres gestacionais e à média de $\mathrm{Hb}$ dos 
682 M.D. SOUZA FILHO et al.

grupos fortificado e não fortificado. Foi considerado um alfa menor que 0,05 como parâmetro de rejeição da hipótese de nulidade $(p<0,05)$. Os dados foram processados no programa Epi Info, versão $6.04 b^{11}$.

A pesquisa seguiu as determinações da Resolução n 196/96 do Conselho Nacional de Saúde ${ }^{12}$ e o prescrito na Convenção de Helsinki13. O estudo foi aprovado pelos Comitês de Ética em Pesquisa da Escola de Enfermagem da Universidade de São Paulo em 2006 (Parecer n 521/06) e da Universidade Federal do Piauí em 2007 (Parecer $n^{\circ}$ 229/07). Todas as gestantes entrevistadas assinaram um termo de consentimento livre e esclarecido antes de sua inclusão na amostra.

\section{RESULTADOS}

As gestantes dos grupos estudados diferiram em relação à situação conjugal e escolaridade $(p<0,05)$, com maior proporção de gestantes sem companheiro e com maior escolaridade no grupo fortificado (Tabela 1).

A distribuição dos valores médios de $\mathrm{Hb}$ segundo o trimestre gestacional e o grupo encontra-se na Tabela 2. Os níveis médios de $\mathrm{Hb}$ aumentaram de $11,7 \mathrm{~g} / \mathrm{dL}, \mathrm{DP}=1,2$ para $12,4 \mathrm{~g} / \mathrm{dL}$, $\mathrm{DP}=1,3$ após a fortificação $(p<0,001)$. No total, o incremento de $0,7 \mathrm{~g} / \mathrm{dL}$, constatado no grupo fortificado, mostrou-se estatisticamente maior $(p<0,001)$. Essa associação foi observada no $1^{\circ} \mathrm{e}$ $2^{\circ}$ trimestres, mas não no $3^{\circ}$ trimestre, possivelmente devido ao menor número de gestantes que iniciaram o pré-natal nesse período.

Apresenta-se na Tabela 3 a prevalência de anemia $(\mathrm{Hb}<11,0 \mathrm{~g} / \mathrm{dL})$ nos grupos não fortificado e fortificado segundo o trimestre gestacional. A prevalência de anemia diminuiu de $27,2 \%$ no grupo não fortificado para $11,5 \%$ no grupo fortificado, redução de $58,0 \%$, que se mostrou estatisticamente significativa $(p<0,000)$. O mesmo se verificou para o segundo e terceiro trimestres $(p<0,000)$, com reduções de $20,5 \%$ para $5,1 \%$ e de $33,0 \%$ para $15,6 \%$, respectivamente.

Considerando que a variação nos níveis de $\mathrm{Hb}$ se inicia no segundo trimestre da gestação, os dados de $\mathrm{Hb}$ das gestantes que se encontravam no primeiro trimestre foram analisados utilizando-se também o ponto de corte de 12,0g/dL, adotado pela OMS ${ }^{1}$ para identificar anemia entre muIheres em idade reprodutiva. A prevalência de anemia passaria para $49 \%$ e $30,3 \%$ para os grupos não fortificado e fortificado, respectivamente (dados não apresentados em tabela).

Tabela 1. Características das gestantes estudadas segundo grupos não fortificado e fortificado. Teresina (PI), 2008.

\begin{tabular}{|c|c|c|c|c|c|c|c|}
\hline \multirow{2}{*}{ Variáveis } & \multicolumn{2}{|c|}{ Não fortificado } & \multicolumn{2}{|c|}{ Fortificado } & \multicolumn{2}{|c|}{ Total } & \multirow{2}{*}{$\begin{array}{c}\text { Teste } \chi^{2} \\
p\end{array}$} \\
\hline & $n$ & $\%$ & $\mathrm{n}$ & $\%$ & $n$ & $\%$ & \\
\hline \multicolumn{8}{|l|}{ Idade (anos) } \\
\hline$<20$ & 83 & 19,4 & 69 & 16,1 & 152 & 17,7 & 0,384 \\
\hline $20 \vdash 35$ & 329 & 77,0 & 339 & 79,3 & 668 & 78,2 & \\
\hline$\geq 35$ & 15 & 3,6 & 19 & 4,6 & 34 & 4,1 & \\
\hline \multicolumn{8}{|l|}{ Situação conjugal } \\
\hline Com companheiro & 311 & 72,8 & 248 & 58,0 & 559 & 65,4 & 0,000 \\
\hline Sem companheiro & 116 & 27,2 & 179 & 42,0 & 295 & 34,6 & \\
\hline \multicolumn{8}{|l|}{ Escolaridade } \\
\hline Fundamental & 212 & 49,7 & 171 & 40,0 & 383 & 44,8 & 0,017 \\
\hline Médio & 191 & 44,7 & 225 & 52,7 & 416 & 48,7 & \\
\hline Superior & 24 & 5,6 & 31 & 7,3 & 55 & 6,5 & \\
\hline \multicolumn{8}{|c|}{ Ocupação fora do lar } \\
\hline Não & 315 & 73,8 & 290 & 68,0 & 605 & 70,9 & 0,059 \\
\hline Sim & 112 & 26,2 & 137 & 32,0 & 249 & 29,1 & \\
\hline
\end{tabular}


Em relação ao consumo alimentar, verificou-se que as carnes faziam parte da prática alimentar da grande maioria das gestantes estudadas, sendo consumidas diariamente ou de uma a quatro vezes por semana por mais de $80,0 \%$ delas. Inclusive vísceras e miúdos eram consumidos por $50,0 \%$ das gestantes, de uma a quatro vezes por semana. Entretanto, quase dois terços

Tabela 2. Médias de Hb segundo trimestre gestacional e grupos não fortificado e fortificado. Teresina (PI), 2008.

\begin{tabular}{|c|c|c|c|c|c|c|c|}
\hline \multirow{3}{*}{$\begin{array}{l}\text { Trimestre } \\
\text { gestacional }\end{array}$} & \multicolumn{6}{|c|}{ Hemoglobina ( $\mathrm{Hb})$} & \multirow{3}{*}{$\begin{array}{c}\text { Teste } t \\
p\end{array}$} \\
\hline & \multicolumn{3}{|c|}{ Não fortificado } & \multicolumn{3}{|c|}{ Fortificado } & \\
\hline & $n$ & M & DP & $n$ & $M$ & DP & \\
\hline $1^{\circ}$ & 200 & 11,9 & $0,9^{a}$ & 175 & 12,6 & $1,5^{\mathbf{b}}$ & $<0,001$ \\
\hline $2^{\circ}$ & 197 & 11,4 & $1,2^{\mathrm{a}}$ & 230 & 12,2 & $1,7^{\mathbf{b}}$ & $<0,001$ \\
\hline $3^{\circ}$ & 30 & 11,5 & $1,2^{\mathrm{a}}$ & 22 & 12,3 & $1,5^{\mathrm{a}}$ & 0,052 \\
\hline Total & 427 & 11,7 & $1,2^{\mathrm{a}}$ & 427 & 12,4 & $1,3^{\mathbf{b}}$ & $<0,001$ \\
\hline
\end{tabular}

Letras iguais não mostram diferença estatisticamente significativa, segundo teste $t(p<0,05)$.

M: média; DP: desvio-padrão.

Tabela 3. Distribuição das gestantes anêmicas $(\mathrm{Hb}<11,0 \mathrm{~g} / \mathrm{dL})$ segundo trimestre gestacional e grupos não fortificado e fortificado. Teresina (PI), 2008.

\begin{tabular}{lccccccc}
\hline Trimestre & \multicolumn{2}{c}{ Não fortificado } & & \multicolumn{2}{c}{ Fortificado } & Teste $\chi^{2}$ \\
\cline { 2 - 3 } gestacional & $n$ & $\%$ & & $n$ & $\%$ & $p$ \\
\hline $1^{\circ}$ & 41 & $20,5^{\mathbf{a}}$ & & 9 & $5,1^{\mathbf{b}}$ & $<0,001$ \\
$2^{\circ}$ & 65 & $33,0^{\mathbf{a}}$ & & 36 & $15,6^{\mathbf{b}}$ & $<0,001$ \\
$3^{\circ}$ & 10 & $33,3^{\mathbf{a}}$ & & 4 & $18,2^{\text {a }}$ & 0,368 \\
\hline Total & 116 & 27,2 & & 49 & 11,5 & $<0,001$ \\
\hline
\end{tabular}

Letras iguais não mostram diferença estatisticamente significativa, segundo teste $\chi^{2}(p<0,05)$. raramente ou nunca consumiam leguminosas (feijão, lentilha, ervilha, grão de bico, soja). Entre os alimentos fortificados, destacou-se o consumo frequente de macarrão $(83,3 \%)$ e preparações com fubá ou farinha de milho $(80,1 \%)$, além de pães, biscoitos e bolos $(60,1 \%)$. O consumo de frutas cítricas (laranja, lima, limão, tangerina, goiaba, caju, cajá e acerola) também era frequente, da mesma forma que a presença de hortaliças de folha verde (couve, espinafre, brócolis, quiabo, repolho e alface) que, no entanto, foram referidos como nunca ou raramente consumidos por mais de um terço das gestantes (Tabela 4).

\section{S C U S S Ã O}

Os resultados evidenciaram que, no período anterior à fortificação das farinhas, a prevalência de anemia entre as gestantes atendidas nos serviços públicos de pré-natal estudados representava um problema de importância epidemiológica moderada (27,2\%). Após a fortificação, o problema passou para a categoria leve $(11,5 \%)$, segundo a classificação da OMS ${ }^{1}$. De acordo com tal classificação, que se baseia na prevalência de anemia em gestantes e pré-escolares, as categorias podem variar de normal a aceitável quando a prevalência de anemia nesses grupos for inferior a 5,0\%; ser considerada leve se estiver entre 5 e 20,0\%; moderada, de 20 a 40,0\%, e grave se a prevalência for maior ou igual a $40,0 \%{ }^{1}$.

Tabela 4. Grupos de alimentos fontes naturais de ferro, facilitadores da absorção do mineral e preparados à base de farinhas fortificadas consumidos por gestantes segundo frequência. Teresina (PI), 2008.

\begin{tabular}{lcccc}
\hline \multirow{2}{*}{ Grupos } & Diariamente & De 1 a 4 vezes por semana & Nunca e raramente \\
\cline { 2 - 3 } Carne bovina & $\%$ & $\%$ & 16,7 \\
Carne de porco e frango & 33,3 & 50,0 & 13,3 \\
Vísceras e miúdo & 6,7 & 80,0 & 46,7 \\
Leguminosas & 3,3 & 50,0 & 63,4 \\
Macarrão & 3,3 & 33,3 & 16,7 \\
Farinha de milho ou fubá & 33,3 & 50,0 & 20,0 \\
Pães, doces e biscoitos & 26,6 & 53,4 & 39,9 \\
Frutas cítricas & 18,3 & 41,8 & 14,0 \\
Hortaliças de folha verde & 56,7 & 29,3 & 36,6 \\
\hline
\end{tabular}


Nos países desenvolvidos, a fortificação de alimentos é utilizada há muito tempo com resultados eficientes no controle das deficiências de micronutrientes, porém a efetivação das medidas tem sido obtida mediante decisões políticas que culminaram no caráter compulsório da fortificação ${ }^{14,15}$.

O Chile controlou a anemia por deficiência de ferro em pré-escolares, escolares e homens adultos. Entre os lactentes, a prevalência diminuiu sensivelmente para cerca de $8 \%$, assim como entre as mulheres em idade fértil. Esse quadro favorável tem sido obtido com programas nacionais de fortificação de alimentos, como a farinha de trigo, fortificada com ferro desde a década de 1950, da mesma forma que o leite do Programa Nacional de Alimentação Complementar, desde 1999. Entretanto, a anemia ainda afeta cerca de $20 \%$ das mulheres no segundo e terceiro trimestres da gestação ${ }^{16}$.

Apesar do aparente sucesso da fortificação de alimentos com ferro, poucas avaliações formais do impacto dessa intervenção têm sido efetivamente desenvolvidas em nível populacional ${ }^{17}$. No Brasil, experimentos com crianças têm evidenciado resultados positivos ${ }^{18-23}$, porém, na perspectiva populacional, os resultados não são tão favoráveis. Estudo de série temporal desenvolvido no sul do Brasil, que avaliou o efeito da fortificação das farinhas anteriormente a essa medida, 12 e 24 meses após sua implantação, não observou efeito dessa intervenção nos níveis de $\mathrm{Hb}$ das crianças estudadas, o que para os autores poderia ser parcialmente explicado pelo baixo consumo de alimentos preparados à base de farinhas e/ou pela baixa biodisponibilidade do ferro adiciona$\mathrm{do}^{24}$.

Em nosso meio, estudos que avaliaram o efeito da fortificação das farinhas com ferro no controle da anemia em gestantes mostraram resultados discordantes a respeito da efetividade da intervenção. Resultados positivos, como o verificado em Teresina (PI), também foram observados por Côrtes ${ }^{25}$ e Santos $^{26}$. Em Brasília, Côrtes ${ }^{25}$ constatou redução significativa de anemia: de 29,0\% no período anterior à fortificação das farinhas para 8,0\% apenas um ano após a implementação da medida $(p<0,001)$. Em uma maternidade social da cidade de São Paulo, Santos ${ }^{26}$ obteve redução menor na prevalência de anemia, de 29,5\% para $20,9 \%$ nos períodos anterior e posterior à fortificação das farinhas, porém ainda assim estatisticamente significativa $(p=0,003)^{26}$.

Outros estudos, no entanto, não observaram mudança nas prevalências de anemia. Em Cuiabá (MT), estudo que avaliou gestantes atendidas em todas as unidades de saúde da família do município constatou prevalências elevadas de anemia tanto no período anterior quanto no posterior à fortificação das farinhas: 25,6\% e 30,6\%, respectivamente ${ }^{27}$. Da mesma forma, estudo que abrangeu todas as unidades básicas de saúde de Maringá (PR) não verificou alteração nas prevalências de anemia, que se mostraram baixas - 11,3\% e $9,4 \%$, respectivamente -, nos períodos anterior e posterior à fortificação das farinhas ${ }^{28}$. Outros dois estudos desenvolvidos em unidades básicas do estado de São Paulo também encontraram prevalências baixas de anemia (8,1\% a 14,3\%), tanto antes quanto depois da implementação da medida de fortificação das farinhas, ou seja, não houve alteração 29,30 .

A comparação dos resultados obtidos em Teresina (PI) com outros estudos parece indicar que nas localidades onde a prevalência de anemia era elevada, os resultados positivos da fortificação das farinhas puderam ser constatados em curto prazo $^{14,15}$. Entretanto, esse padrão não foi observado em Cuiabá27.

Ao aumentar a sensibilidade do diagnóstico para mulheres no primeiro trimestre da gestação, com a utilização do valor crítico recomendado para identificar anemia em mulheres em idade reprodutiva $(12,0 \mathrm{~g} / \mathrm{dL})$, o quadro de anemia obtido foi bastante diverso, porém ainda assim com evidências de melhoria no quadro, que passou de importância epidemiológica grave no grupo não fortificado para moderada após a fortificação. Vale destacar que a prevalência de 30,3\% verificada em gestantes do primeiro trimestre do grupo 
fortificado foi similar ao obtido pela Pesquisa Nacional de Demografia e Saúde da Criança e da Mulher (PNDS) para a população brasileira de mulheres em idade reprodutiva $(29,4 \%)^{8}$. É importante lembrar que os dados dessa pesquisa foram colhidos em 2006, quando a fortificação tinha um ano de implantação, e que essa é uma intervenção cujos resultados efetivos são esperados em longo prazo.

Dados da PNDS ${ }^{8}$ indicaram que, no Nordeste, região onde se localiza Teresina (PI), a anemia entre as mulheres em idade reprodutiva atingia $39,1 \%$. Esse resultado reitera a importância de medidas de intervenção para o controle dessa deficiência nutricional, tal como a obrigatoriedade da fortificação das farinhas de trigo e milho com ferro, que integra a Política Nacional de Alimentação e Nutrição ${ }^{31}$. Justifica-se também o desenvolvimento de investigações que avaliem o efeito de intervenções, como o presente estudo, que retratou a prevalência de anemia em um grupo vulnerável, antes e depois da fortificação das farinhas.

Apesar da importante queda na prevalência de anemia, decorrente da elevação significativa nos valores de $\mathrm{Hb}$ após a fortificação das farinhas, os resultados obtidos devem ser interpretados no contexto das potenciais limitações de um estudo transversal, mesmo que se tenha avaliado dois períodos: um anterior à fortificação e outro pelo menos um ano depois dela.
Considerando que a ocorrência da anemia é socialmente determinada, o fato de uma proporção significativamente maior de gestantes do grupo fortificado não ter companheiro poderia indicar pior situação social e consequentemente maior probabilidade de desenvolver anemia. Entretanto, proporção mais elevada de gestantes desse mesmo grupo apresentou maior escolaridade, o que pode ter compensado tal situação desfavorável ${ }^{32}$. Certamente, assim como essas variáveis analisadas podem ter interferido nos resultados, muitas outras variáveis não investigadas também podem ter contribuído para o aumento das médias de $\mathrm{Hb}$ e redução da anemia.

Há que se destacar, no entanto, que a avaliação da prática alimentar mostrou que, apesar da baixa renda, as gestantes atendidas nos serviços públicos de saúde de Teresina (PI) apresentavam consumo frequente de carnes, fonte de ferro de melhor biodisponibilidade, e de frutas cítricas, fonte de vitamina $\mathrm{C}$, ativador da biodisponibilidade do mineral ${ }^{33}$.

Verificou-se, também, consumo frequente de alimentos preparados à base de farinha de trigo e milho. Em Brasília, no entanto, mais de $90 \%$ das gestantes estudadas referiram raramente consumir preparações com farinha de milho ${ }^{34}$.

Com vistas a um melhor entendimento da participação da farinha de trigo na alimentação dos brasileiros, analisou-se o gasto com farinhas, féculas e massas, a partir de dados da Pesquisa de Orçamento Familiar (POF) ${ }^{35}$, uma vez que os

Quadro 1. Estimativa de consumo de derivados de farinha de trigo (pão, massas e bolachas) no total da população brasileira e naquelas com renda <2 salários-mínimos, segundo região. Brasil, 2004.

\begin{tabular}{lccccc}
\hline & \multicolumn{2}{c}{ Consumo de farinha de trigo (g/dia) } & & \multicolumn{2}{c}{ Ferro biodisponível (mg) } \\
\cline { 2 - 3 } \cline { 5 - 6 } Região & População total & $\begin{array}{c}\text { População com renda } \\
<2 \text { salários-mínimos }\end{array}$ & & População total & $\begin{array}{c}\text { População com renda } \\
<2 \text { salários-mínimos }\end{array}$ \\
\hline Norte & 51,0 & 13,2 & 1,73 & 0,35 \\
Nordeste & 66,0 & 16,3 & 2,24 & 0,55 \\
Sudeste & 72,3 & 18,2 & & 2,46 & 0,61 \\
Sul & 70,4 & 17,4 & 2,39 & 0,60 \\
Centro-Oeste & 45,5 & 11,4 & & 1,55 & 0,39 \\
\hline Brasil & 66,8 & 16,2 & 2,27 & 0,54 \\
\hline
\end{tabular}

Fonte: Instituto Brasileiro de Geografia e Estatística ${ }^{35}$. 
686 | M.D. SOUZA FILHO et al.

resultados dessa pesquisa fornecem dados indiretos do consumo alimentar, apesar de avaliarem o gasto com a compra de gêneros alimentícios.

O Quadro 1, que apresenta uma estimativa do consumo diário per capita de farinhas de trigo e do ferro biodisponível para a população geral e para aquelas com renda inferior a dois salários-mínimos, mostra que o consumo de farinha de trigo é menor entre a população de mais baixa renda.

Possivelmente as gestantes estudadas em Teresina (PI) apresentavam uma condição diferenciada de alimentação, com consumo bastante frequente de fontes naturais de ferro (carnes e vegetais de folha verde-escuro) e de facilitadores de sua absorção (frutas cítricas), além de alimentos fortificados.

\section{O N CLUSÃ O}

Os resultados evidenciaram aumento nos níveis de $\mathrm{Hb}$, com incremento de 0,7g/dL no grupo fortificado e redução significativa de quase $60 \%$ na prevalência de anemia, pelo menos um ano após a efetiva implantação da fortificação das farinhas.

Observou-se também que a maioria das gestantes apresentava consumo frequente de alimentos fontes naturais de ferro e de facilitadores da absorção do mineral, além de alimentos fortificados à base de farinha de trigo e de milho, que podem ter contribuído para tal resultado favorável observado nos serviços públicos de saúde estudados.

Por fim, há que se considerar que, apesar dos resultados promissores encontrados em Teresina (PI), que sugerem efeito positivo da fortificação das farinhas no controle da anemia, o estudo não permite atribuir tal resultado apenas à implantação dessa estratégia. Entretanto, como se trata de uma medida permanente que beneficia toda a população, espera-se que, em longo prazo, os indivíduos sejam capazes de apresentar uma reserva de ferro suficiente para o enfrentamento de processos fisiológicos normais de crescimento e de concepção, tornando-se livres dos riscos decorrentes da deficiência marcial do mineral.

\section{O LABORADORES}

M.D. SOUZA FILHO participou da concepção, do planejamento, da coleta, da análise, da interpretação dos dados e da elaboração do artigo. C.V.X. DAMASCENO participou da coleta e da elaboração do banco de dados. S.C. SZARFARC e E. FUJIMORI participaram da concepção, do planejamento da investigação e da revisão crítica do manuscrito. M.A.M. ARAÚJO participou da concepção, do planejamento, da análise estatística e da interpretação dos dados do artigo. R.S.R. MOREIRA-ARAÚJO orientou a concepção, o planejamento, a análise e a interpretação dos dados e participou da elaboração e da revisão do manuscrito.

\section{REFERÊ N CIAS}

1. World Health Organization. Iron deficiency anaemia. Assessment, preventing, and control. A guide for programme managers. Geneva: WHO; 2001 [cited 2009 Nov 21]. Available from: <http:// whqlibdoc.who.int/hq/2001/WHO_NHD_01.3. pdf>.

2. Batista Filho M, Souza Al, Bresani CC. Anemia como problema de saúde pública: uma realidade atual. Ciênc Saúde Coletiva. 2008; 13(6):1917-22. doi: 10.1590/S1413-81232008000600027.

3. Souza Al, Batista Filho M, Ferreira LOC. Alterações hematológicas e gravidez. Rev Bras Hemat Hemoter 2002; 24(1):29-36. doi: 10.1590/\$1516-84842002 000100006 .

4. Fujimori E, Laurenti D, Nunez de Cassana LM, Oliveira IMV, Szarfarc SC. Anemia e deficiência de ferro em gestantes adolescentes. Rev Nutr. 2000; 13(3):177-84. doi: 10.1590/S1415-52732000000 300004.

5. Barón MA, Solano L, Pena E, Sánchez A, Del Real S. Estado de las reservas de hierro al inicio del embarazo. Invest Clín. 2005; 46(2):121-30.

6. International Nutritional Anemia Consultative Group. Guidelines for eradication of iron deficiency. New York: INACG; 1977.

7. Brasil. Resolução RDC $n^{\circ} 344$, de 13 de dezembro de 2002. Áreas de atuação. Alimentos. Legislação específica da área por assunto. Regulamentos 
técnicos por assunto. Farinhas de trigo e/ou milho fortificadas com ferro. [acesso 2010 jan 21]. Disponível em: <http://e-legis.bvs.br>.

8. Brasil. Ministério da Saúde. Pesquisa nacional de demografia e saúde da criança e da mulher PNDS 2006: dimensões do processo reprodutivo e da saúde da criança. Brasília: MS; 2009.

9. Brasil. Ministério da Saúde. Secretaria de Atenção à Saúde. Departamento de Ações Programáticas Estratégicas. Área Técnica de Saúde da Mulher. Pré-natal e puerpério: atenção qualificada e humanizada. Brasília: MS; 2006. Manual Técnico. Série A. Normas e Manuais Técnicos.

10. Ribeiro AC, Sávio KEO, Rodrigues MLCF, Costa THM, Schmitz BAS. Validação de um questionário de freqüência de consumo alimentar para população adulta. Rev Nutr. 2006; 19(5): 553-62. doi: 10.1590/S1415-52732006000500003.

11. Dean AG, Dean JA, Coulombier D, Brendel KA, Smith DC, Burton AH, et al. Epi Info, version 6: a word processing, database, and statistics program for epidemiology on microcomputers. Atlanta, Georgia: Centers for Disease Control and Prevention; 1994.

12. Brasil. Ministério da Saúde. Resolução 196/96 do Conselho Nacional de Saúde sobre Diretrizes e Normas Regulamentadoras de Pesquisa envolvendo seres humanos. Diário Oficial de União. 199610 out.

13. World Medical Association. Declaration of Helsinki. Ethical Principles for Medical Research Involving Human Subjects. Proceedings of the $59^{\text {th }}$ WMA General Assebly, Seoul, Korea, 2008.

14. Backstrand JR. The history and future of food fortification in the United Sates: a public health perspective. Nutr Rev. 2002; 60(1):15-26. doi: 10.1301/002966402760240390.

15. Baltussen R, Knai C, Sharan M. Iron fortification and iron supplementation are cost-effective interventions to reduce iron deficiency in four subregions of the world. J Nutr. 2004; 134(10): 2678-84.

16. Olivares M, Pizarro F, Hertrampf E, Walter T, Arredondo M, Letelier A. Fortificación de alimentos con hierro en Chile. Rev Chil Nutr. 2000; 27(3): 340-4.

17. Allen L, Benoist B, Dary O, Hurrell R, editors. Guidelines on food fortification with micronutrients. Geneva: Organization of the United Nations; 2006.

18. Moreira-Araújo RSR, Araújo MAM, Silva AMS, Carvalho CMR, Arêas JAG. Impacto de salgadinho de alto valor nutritivo na situação nutricional de crianças de creches municipais de Teresina-PI. Nutrire Rev Soc Bras Aliment Nutr. 2002; 23(1): 7-21.
19. Silva MR, Castro TG, Costa NMB, Ferreira CLLF, Franceschini SCC, Leal PFG, et al. Efeito de uma bebida láctea fermentada e fortificada com ferro sobre o estado nutricional de ferroem pré-escolares. Viçosa-MG. Nutrire: Rev Soc Bras Aliment Nutr. 2002; 23(1):23-32.

20. Tuma RB, Yuyama LKO, Aguiar JPL, Marques HO. Impacto da farinha de mandioca fortificada com ferro aminoácido quelato no nível de hemoglobina de pré-escolares. Rev Nutr. 2003; 16(1):29-39. doi: 10.1590/S1415-52732003000100004.

21. Marchi RP, Szarfarc SC, Rodrigues JEG. Consumo de arroz fortificado com ferro na profilaxia da deciência do mineral. Nutrire: Rev Soc Bras Aliment Nutr. 2004; 28(1): 53-64.

22. Moreira-Araujo RSR, Araújo MAM, Arêas JAG. Fortified food made by extrusion of a mixture of chickpea, corn and bovine lung controls irondeficiency anaemia in preschool children. Food Chemistry. 2008; 107(1):158-64.

23. Costa CA, Machado EH, Colli C, Latorre WC, Szarfarc SC. Anemia em pré-escolares atendidos em creches de São Paulo (SP): perspectivas decorrentes da forticação das farinhas de trigo e de milho. Nutrire: Rev Soc Bras Aliment Nutr. 2009; 34(1):59-74.

24. Assunção MCF, Santos IS, Barros AJD, Gigante DP, Victora CG. Efeito da fortificação de farinhas com ferro sobre anemia em pré-escolares, Pelotas, RS. Rev Saúde Pública. 2007; 41(4):539-48. doi: 10.1590/S0034-89102006005000031.

25. Côrtes MH. Impacto da fortificação das farinhas com ferro nos níveis de hemoglobina das gestantes atendidas pelo pré-natal do Hospital Universitário de Brasília [dissertação]. Brasília: Universidade de Brasília; 2006 [acesso 2010 jan 21]. Disponível em: $<$ http://bdtd.bce.unb.br/tedesimplificado/tde_ busca/arquivo. php?codArquivo=146> .

26. Santos AU. Prevalência de anemia em gestantes atendidas em uma maternidade social: antes e após a fortificação das farinhas com ferro [dissertação]. São Paulo: Universidade de São Paulo; 2009 [acesso 2010 jan 21]. Disponível em: <http://www.teses. usp.br/teses/disponiveis/7/7132/tde-22062009-12 4452/>.

27. Porto ES. Impacto da fortificação das farinhas de trigo e de milho com ferro e ácido fólico na concentração de hemoglobina de gestantes [dissertação]. Cuiabá: Universidade Federal de Mato Grosso; 2007.

28. Araújo CRMA. Concentração de hemoglobina em gestantes atendidas em serviços de saúde do sul do Brasil, antes e após a ingestão de ferro em ali- 
mentos fortificados [dissertação]. Maringá: Universidade Estadual de Maringá; 2007 [acesso 2010 jan 21]. Disponível em: <http://www.pse.uem.br/ index_arquivos/dissert-claudia.pdf>.

29. Sato APS, Fujimori E, Szarfarc SC, Sato JR, Bonadio IC. Prevalência de anemia em gestantes e a fortificação de farinhas com ferro. Texto Contexto Enferm. 2008;17(3):481-9. doi: 10.1590/S0104-070720 08000300008 .

30. Abreu LC. Impacto da fortificação das farinhas com ferro no controle da anemia em gestantes: estudo em um serviço público de saúde do município de São Bernardo do Campo [dissertação]. São Paulo: Universidade de São Paulo; 2009 [acesso 2010 jan 21]. Disponível em: <http://www.teses.usp.br/teses/ disponiveis/6/6138/tde-28082009-173919/>

31. Brasil. Ministério da Saúde. Secretaria de Atenção à Saúde. Política nacional de alimentação e nutrição. 2a ed. Brasília: MS; 2003.

32. Vitolo MR, Boscaini C, Bortolini GA. Baixa escolaridade como fator limitante para o combate à anemia entre gestantes. Rev Bras Ginecol Obstet. 2006;
28(6):331-9. doi: 10.1590/S0100-720320060006 00003.

33. Pereira RC, Diniz AS, Ferreira LOC. New findings on iron absorption conditioning factors. Rev Bras Saúde Mater Infant. 2004; 4(3): 241-8. doi: 10.15 90/S1519-38292004000300003.

34. Vasconcelos IAL, Cortes MH, Coitinho DC. Alimentos sujeitos à fortificação compulsória com ferro: um estudo com gestantes. Rev Nutr. 2008; 21(2):149-60. doi: 10.1590/\$1415-52732008000 200003.

35. Instituto Brasileiro de Geografia e Estatistica. Pesquisa de orçamentos familiares 2002-2003. Primeiros resultados. Brasil e Grandes Regiões. Rio de Janeiro: IBGR; 2004 [acesso 2008 nov 21]. Disponível em: <http://www.ibge.gov.br/home/ estatistica/populacao/condicaodevida/pof/2002/ pof200220032aed.pdf>.

Recebido em: 6/5/2010

Versão final reapresentada em: 22/3/2011 Aprovado em: 8/7/2011 